\author{
Allan Sandage \\ Center for Astrophysical Sciences \\ Department of Physics and Astronomy \\ The Johns Hopkins University \\ Baltimore, Maryland 21218, USA
}

\begin{abstract}
The history of modern cosmology has been divided into two periods. The primary discovery phase extended from 1920 to $\sim 1950$ when most of the classical tests were developed. The subsequent consolidation period from 1950 to $\sim 1980$ saw a deeper understanding of the tests and the introduction of concepts of stellar populations that led to estimates of evolutionary trends with look-back time. The only test not affected by evolutionary effects is the comparison of the Hubble time, $\mathrm{H}^{-1}$, with independent estimates of the age of the Universe. The $\mathrm{N}(\mathrm{m}), \mathrm{m}(\mathrm{z}), \theta(\mathrm{z}), \theta(\mathrm{m})$, and $\mathrm{H}_{\mathrm{o}} \mathrm{T}_{\mathrm{o}}\left(\mathrm{q}_{0}, \Lambda\right)$ tests are reviewed as they have been discussed in the archive literature. The problem of observational bias is emphasized, as is the difficult recent search for secular evolutionary effects. Current problems which have prospects for solutions in the next decades are set out in the final section.
\end{abstract}

\title{
PREVIEW
}

Cosmology is now a dominant field of modern astronomy, carrying a principal theme in its own right, but also being the background motivation for much of the present work in even traditional astronomical fields from astrometry, to stellar atmospheres and interiors, to galactic structure, and the interstellar medium. The present cosmological thrust is to devise methods for calibrating various cosmological parameters such as $\mathrm{H}$, the time scale, the $3^{\circ} \mathrm{K}$ flux, etc., but ultimately to reach back to the origins of everything from stars, to galaxies, to the Universe itself; the borders of cosmology and cosmogony here being blurred.

The connection of cosmology to nearly all other parts of astronomy, and the fact that a good fraction of the young astronomers now take cosmology to be their subject, has not always been. As late as 1938, the last year before the war, there were probably less than 30 cosmologists in the world, and of these perhaps only $\sim 15$ had the status of builders of the foundations. Today there are $\sim 1000$ cosmologists, of which $\sim 30 \%$ are at this conference. This rapid growth corresponds to a doubling time of $\sim 10$ years, or a $7 \%$ growth per year. At this rate, in 
100 years there will be a million cosmologists, giving hope that the cosmological problem can, in fact, be solved. At the moment it is not.

We know the questions now better than at any previous time, and, fortunately, the powerful instrumentation, either just operating or on the horizon to be built, signals the start of the observational survey effort required to truly sample the World. It is in this sense that our conference here, I think, marks the final beginning of the subject.

Looking over the program, we shall see discussions this week of the enormous progress and the high expectations for the massive data collection that is required. To be sure, there has been much exploratory work over the past half century which has led us to this starting place. My plan for this introduction is to review the past so as to trace the origins of the major themes we shall hear repeatedly throughout the meeting. In one way or another, each speaker will return to the same set of basic topics, centered on the four classical cosmological tests. The history of the development of these tests forms the organizing frame of this introduction.

It is convenient to divide the past into two segments, separated at 1950 with the beginning of observations with the Hale 200-inch reflector. This divides the time from 1920 to 1980 into two equal intervals - the foundational period from 1920 to 1950 when the elements of the subject were laid down, and 1950 to 1980 which was a period of consolidation, punctuated, to be sure, by the unexpected major discoveries of radio astronomy and the $3^{\circ} \mathrm{K}$ radiation.

The last 30 years have been a period of understanding the tests, set out early, primarily by Hubble, but sharpened by the interpretative insight of Tolman and Robertson. At the very start of the Hale telescope period in 1950, high simplistic hopes were held that only a few years would be required to find such things as the expansion rate and the deceleration, to actually measure the curvature of space by Gauss's experiment with surfaces, generalized to volumes in Riemannian manifolds, and to test if the expansion is real or if the redshift is from some unknown new principle. But at every step, each of the tests has been blocked or delayed until a great deal had to be understood about the details of the test objects. This now has taken cosmologists about 30 years to accomplish.

Hubble was primarily interested in the large scale geometry of space. He looked upon galaxies as markers, either of the geometry directly, or as a means to measure the deceleration of the redshift with time (i.e., to determine the "second term" in the redshift - distance relation). This second term - $\mathrm{q}_{\mathrm{o}} \mathrm{z}-$ leads to the space curvature through the postulate of general relativity that the components $g_{i j}$ of the
metric tensor are related to the mean mass density, which itself controls the deceleration.

Baade, Hubble's colleague at Mount Wilson, was always opposed to this direct path. Each of the primary tests [the $m(z), N(m), \theta(z)$, and $\mathrm{SB}(z)$ relations] uses some measured parameter of the galaxy such as apparent magnitude, angular diameter, or surface brightness. These obviously are subject to changes with time, and therefore with redshift in an evolving Universe. Baade's dictum was always 
"You must understand the galaxies before you can get the geometry right."

Hubble clearly understood this, but rather than be stopped because this part of his subject was 30 years before its time, he pushed ahead with an abandonment known to pioneers in any milieu who try to reach Everest without proper equipment. Some, indeed, succeed. Without Hubble's attempt, the foundations of our subject would not now be in place for us and for the next generation.

Meanwhile, while Hubble was emphasizing both the redshift magnitude relation and the galaxy number counts as ways to determine the spatial curvature, Baade was indeed trying to understand the galaxies themselves. From the late 1930's until 1950 two parallel, nearly nonintersecting flows in cosmology were occurring in Pasadena - one using galaxies as markers; the other with galaxies as keepers of various stellar populations. This second avenue - Baade's - hardly existed in its present recognized form until its beginnings in $\sim 1940$. The modern ideas of stellar evolution, age dating the stars, the change of the HR diagram with time, color and luminosity evolution of the total light, etc., is a product of the decade from 1950 to 1960 in its foundations, and 1960 to 1970 in the working out of its details. Hence, Baade's dictum could not have led to any reasonable actions until quite recently. It has only been a few years now since these two great separate streams of inquiry met to form the modern formulation of the work.

This review is centered on the tests. I propose to introduce each test as Hubble first set it out, to discuss how each was treated and what we learned in the consolidation period, and finally to look at what is possible concerning the completion of each test in the future with the new instrumentation.

\section{THE TESTS}

a) The $N(m)$ count distribution was thought to be a direct attack on the experimental geometry following the method of Gauss for surfaces. In curved space the circumference of a small circle of radius $d$ on, for example, the surface of a sphere of radius $R$ is not $2 \pi d$ but is

$$
L(d)=2 \pi d\left(1-\frac{1 d^{2}}{6 R^{2}}+---\right),
$$

providing a means to determine the radius of curvature $R$ by measuring $L$ and $d$ along the surface of the sphere.

Likewise, the area of a spherical cap of radius $d$ on the surface is not $\Pi d^{2}$ as in Euclidean space, but is

$$
A(d)=\pi d^{2}\left(1-\frac{1}{12} \frac{d^{2}}{R^{2}}+---\right),
$$

again affording an experimental determination of $\mathrm{R}$ by measuring the deviation of the measured area from $\pi d^{2}$. 
These two examples are easy to visualize because the curved surface is the familiar two dimensional space of a sphere which is embedded in a Euclidean three dimensional enveloping manifold. The visualization of three dimensional Riemannian space is more difficult because it is outside direct experience. Nevertheless, it forms the essence of Einstein's replacement of action at a distance (force without contact via a field) where, in the presence of a mass, a test particle moves along a straight path in the warp of curved space rather than on a curved path in straight space. By 1930 the idea of curved space was commonplace enough to attempt a direct measurement of volumes, similar to the earlier experiment by $\mathrm{K}$. Schwarzschild on stellar parallaxes.

In the presence of a three dimensional Gaussian spatial curvature of strength $\mathrm{k} / \mathrm{R}^{2}$, volumes out to a measured "distance" (properly defined) do not increase as $4 \pi \mathrm{d}^{3}$ but rather as

$$
V(d)=\frac{4}{3} \pi d^{3}\left(1-\frac{1}{5} \frac{d^{2}}{R^{2}}+---\right)
$$

again affording a test if proper volumes could be measured.

Hubble proposed galaxy counts to do that, using apparent magnitudes as indicators of distance in the mean, and determining the number, $\mathrm{N}(\mathrm{m})$, of galaxies brighter than apparent magnitude $\mathrm{m}$.

After correcting the apparent magnitudes for the effects of redshift, Hubble assumed that the correct distance to use was a luminosity distance based on

$$
\ell \sim \frac{L}{\pi d^{2}(1+z)^{2}}
$$

if the redshift $z$ was a true expansion, or

$$
\ell=\frac{L}{4 \pi d^{2}(1+z)}
$$

if the redshift was not due to expansion, where $\ell$ and $L$ are the apparent and absolute luminosities over a fixed proper wavelength band pass, correcting the observed $\ell$ for the selective $K$ term due to the redshift of the spectrum through the band pass. (He forgot the stretching factor of $1+z$ in the bandpass due to redshift in his total $\mathrm{K}$ term).

One can show that, given any non-divergent distribution of absolute luminosities $\phi(\mathrm{L})$, i.e. the luminosity function, an integration over $\phi(L)$ for the Euclidean case of $R=\infty$ (in the closed analytical form of equation 3 rather than a series expansion), gives

$$
\mathrm{N}(\mathrm{m}) \sim 10^{0.6 \mathrm{~m}}
$$

for all $\mathrm{m}$. The spatial curvature flattens the $\mathrm{N}(\mathrm{m})$ curve in a way which Hubble expressed as

$$
\log N(m)=\text { const }+0.6(m-\Delta m),
$$

where $\Delta \mathrm{m}$ is a function of distance (i.e. redshift) that depends on the 
spatial curvature $R$. His task was to determine the $\Delta m=f(z)$ function and to infer the spatial curvature from it using the closed form of equation (3), assuming either equation (4) or equation (5).

His data and analysis (Hubble 1936) are shown in Figure 1 here, taken from his Figure 16 in The Realm of the Nebulae, together with the caption he gave the diagram.

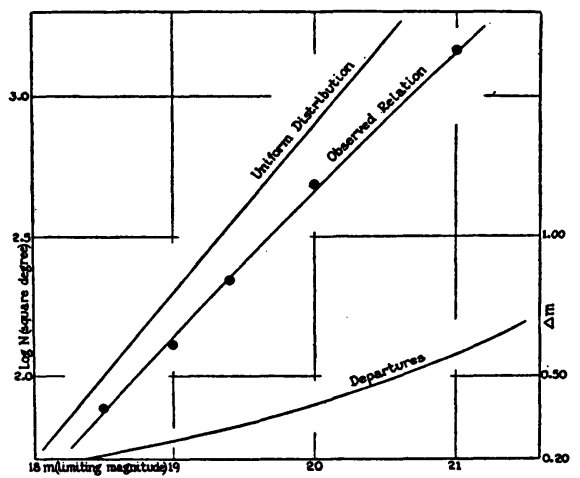

Fig. 16. Apparent Distribution of Nebula in Depth.

Each point on the Observed Relation represents the average number. (actually $\log N$ ) of nebule per square degree which are equal to, or brighter than, a particular apparent magnitude, as determined by an entire survey. The line through the points (Observed Relation) is a least square solution in the form, $\log N=0.6(m-\Delta m)+$ constant, derived from the assumption that $\Delta m$ is a linear function of distance.

In the vicinity of the galactic system, $\Delta m$ should be negligible, and the $\mathrm{Ob}$. served Relation should coincide with a Uniform Distribution indicated by the straight line. As the surveys are extended to greater distances (fainter limiting magnitudes), $\Delta m$ increases, and the Observed Relation departs from the line representing Uniform Distribution. The departures, $\Delta m$ (horizonal displacements between the two lines), are plotted against $m$ (limiting magnitudes of the surveys) in the lowest curve. The departures are interpreted as effects of red-shifts.
Fig. 1 Hubble's (1936) final analysis of his count data as a function of blue magnitude, corrected according to his precepts of the $\mathrm{K}$ correction. His caption from Realm of the Nebulae is reprinted.

His conclusion from the analysis was that the deviation of the observed $\log \mathrm{N}(\mathrm{m})$ curve from a slope of 0.6 was so great that either $R$ was very small and that curved space had indeed been found, or that equation (5) was correct and that the redshift was not due to expansion.

We know now that he could not have found the answer by this route, even in principle because the theory of the redshift - distance relation for any spatial curvature had not yet been worked out. He assumed that the $d$ to be used in equations (4) or (5) was simply proportional to the redshift $z$ for all geometries (i.e. for an value of $R$ ). However, the correct distance to use (Mattig 1958, Sandage 1961a,) is known to be

$$
r=\frac{c}{R_{0} H_{0} q_{o}^{2}(1+z)}\left\{q_{o} z+\left(q_{o}-1\right)\left[-1+\left(1+2 q_{o} z\right)^{1 / 2}\right]\right\}
$$

which differs from

$$
\mathrm{d}=\frac{\mathrm{cz}}{\mathrm{R}_{\mathrm{O}} \mathrm{H}_{\mathrm{O}}}
$$


assumed by Hubble (note that $d$ in eqs. 4 and 5 is the same as $R r(1+z)$ here). The correct equation for the volume contained out to the comoving coordinate $r$ is

$$
V(r)=\frac{4 \pi R^{3} r^{3}}{3}\left[\frac{3}{2} \frac{\sin ^{-1} r}{r^{3}} \frac{3}{2} r^{2}\left(1-r^{2}\right)^{1 / 2}\right]
$$

which can be put in terms of the observed redshift $z$ by substituting equation (8) in (10). A series approximation, correct to order $z$ in the correction term is

$$
V(z)=\frac{4 \pi z^{3}}{3 H_{0}^{3}}\left[1-\frac{3}{2}\left(1-q_{o}\right) z+---\right] .
$$

This can be expressed in apparent luminosity by using equation (4) to finally give the predicted $N(m)$ relation for different values of $q_{0}$ which is the intrinsic geometry parameter, related to the expansion rate $\mathrm{H}_{\mathrm{O}}$, and the spatial curvature $\mathrm{R}_{\mathrm{O}}$ by

$$
\frac{\mathrm{kc}^{2}}{\mathrm{R}_{\mathrm{O}}^{2}}=\mathrm{H}_{\mathrm{O}}^{2}\left(2 \mathrm{q}_{\mathrm{O}}^{-1}\right) \text {. }
$$

When this is done to produce the predicted $\mathrm{N}(\mathrm{m})$ relation, it turns out that $N(m)$ is independent of $q$ to first order in $z$ entering only at order $\mathrm{z}^{2}$ (Mattig 1959, eq. 9; Sandage 1961a; Brown and Tinsley 1974). Only at very high redshifts is the spatial curvature effect felt. Hence the $N(m)$ test is quite insensitive to $q_{0}$ and the test essentially fails even if there would be no luminosity evolution of the galaxies in the look back time.

On the contrary, $N(z)$ rather than $N(m)$ is sensitive to $q$ to first order in the correction term, as shown by equation (11), and this will be the form of the count test to find $q_{0}$ in the future by counting

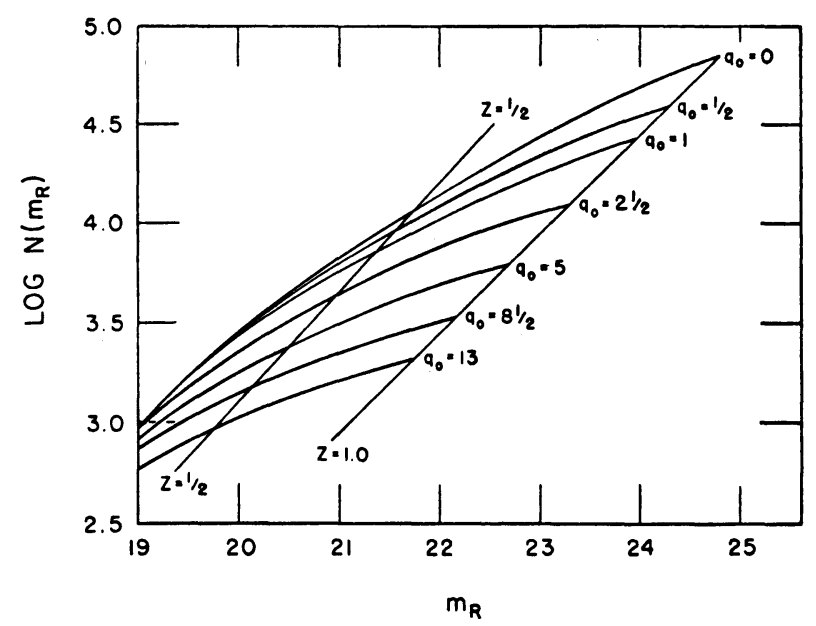

Fig. 2 Predicted $\mathrm{N}\left(\mathrm{m}, \mathrm{q}_{\mathrm{o}}\right)$ from the standard Friedman model using fully corrected red magnitudes $\mathrm{m}_{\mathbf{r}}$. 
objects in a complete sample to a given $z$ (see the review by Sargent in this volume of an application of the test using $\mathrm{L}$ absorption lines in the Lyman forest of quasar spectra where line of sight distances are used rather than volumes).

The insensitivity of the $\mathrm{N}(\mathrm{m})$ test to variations in $\mathrm{q}_{0}$ for $z \lesssim 1$ is shown by idealized calculations made as if $\phi(L)=\delta$ (i.e. no dispersion in L) (Sandage 1961a, Figure 5, shown as Figure 2 here).

Reasonable values of $\mathrm{q}_{\mathrm{o}}$ are between 0 and $1 / 2$, giving very little handle for the $\mathrm{N}(\mathrm{m})$ test according to Figure 2 . The actual difference in the family of curves is even less than shown in the diagram when an integration over $\phi(L)$ is made.

The $N(m)$ test has been applied in recent years to test primarily luminosity evolution of the galaxies rather than intrinsic geometry. Counts by Karachentsev and Kopylov (1978), Kron (1980), Tyson and Jarvis (1979), and Peterson et al. (1979) are examples. Figure 3 shows the final analysis of Peterson et al. (their Figure 4), using proper integrations over luminosity functions for different galaxy types. A model for galaxy luminosity evolution has also been folded in, being the major effect compared with differences of $\mathrm{q}_{\mathrm{o}}$.

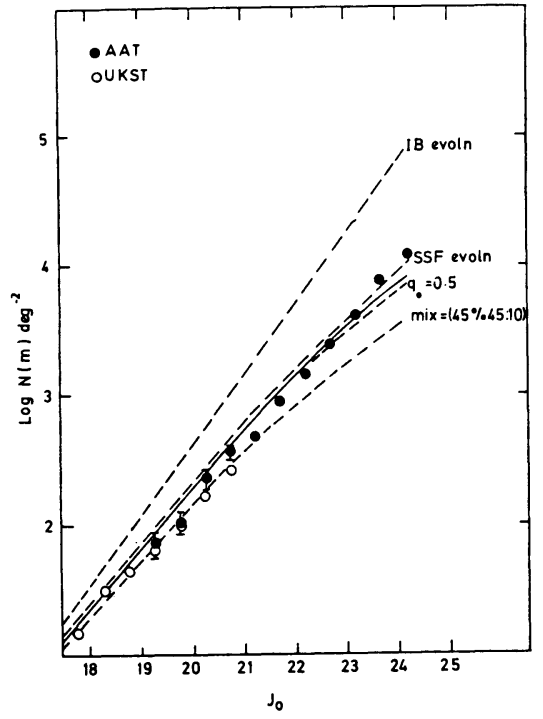

Fig.3 Observed $\mathrm{N}(\mathrm{m})$ from Peterson et al. (1979, their Fig. 4). Various calculated theoretical curves are shown.

The conclusion from Figure 3 is that the $\mathrm{N}(\mathrm{m})$ distribution is very close to what is predicted by the standard model, but that it tells us nothing about the intrinsic geometry; its main use is to test models of galaxy luminosity evolution, on the assumption that the Friedman standard model is correct.

During this conference we will hear of new $N(\mathrm{~m})$ and especially $\mathrm{N}(\mathrm{z})$ data from several different experiments (e.g. Yee, Loh, Tytler and also Murdock, et al. 1986 in Sargent) in attempts to find $q_{0}$.

b) the $\mathrm{m}(\mathrm{z})$ or Hubble diagram test is the most famous of the relations. Hubble's first formulation of the linear redshift-distance relation in 1929 is shown in Figure 4, together with his figure caption from Figure 9 of Realm of the Nebulae. 


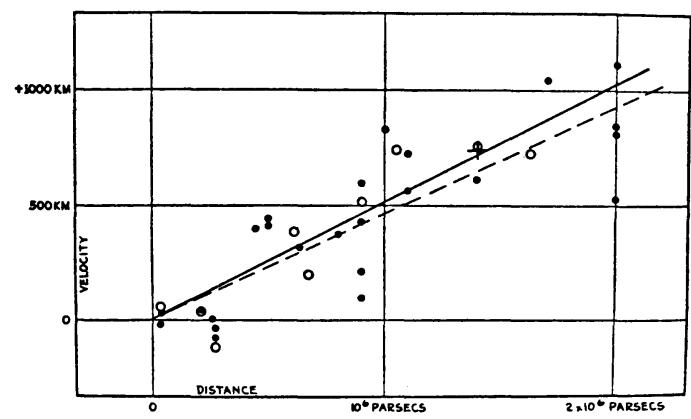

Fig. 9. The Formulation of the Velocity-Distance Relation.

The radial velocities (in $\mathrm{km} / \mathrm{sec}$.), corrected for solar motion, are plotted against distances (in parsecs) estimated from involved stars and, in the case of the Virgo cluster (represented by the four most distant nebula), from the mean luminosity of all nebulæe in the cluster. The black disks and full line represent a solution for the solar motion using the nebula individually; the circles and dashed line, a solution combining the nebulæ into groups.
Fig. 4 Hubble's original 1929

discovery plot of the velocitydistance relation, reproduced from Figure 9 of Realm of the Nebulae.

In his last discussion, which was the Darwin Lecture, Hubble (1953) used the $\mathrm{m}(\mathrm{z})$ observations of brightest cluster galaxies, measured in the first years of the Hale telescope operation. He fit the preliminary data well with an $m_{c} \alpha 5 \log z$ relation, where $m_{c}$ was the apparent magnitude corrected by only one factor of $1+z^{c}$ as in equation( 5), plus that part of the $K$ term which accounts for the shifting of the non-flat galaxy spectrum through the stationary band pass of the detector (but again he forgot that $1+z$ passband stretching term due to the redshift). He discussed the data as if $\log \mathrm{z} \alpha 0.2 \mathrm{~m}$ represented the true linear velocity-distance relation, and that deviătions from it would indicate a change of $\mathrm{z}$ with time (i.e. acceleration or deceleration). He stated that if the additional $1+z$ term (of equation 4 ) was applied to the bolometric magnitude correction, then the upward deviation (i.e. a brighter $\mathrm{m}$ ) of the resulting $\mathrm{m}(\mathrm{z})$ function relative to the $\mathrm{z} \sim 0.2 \mathrm{~m}$ "linear case" would give a non-linear velocity-"distance" relation in the sense of an accelerated expansion. (italics are Hubble's words).

However, Hubble's supposition that $z \sim 0.2 \mathrm{~m}_{\mathrm{c}}$ is the nondecelerated case is based on equation (9), which, ${ }^{c}$ although it is the intuitive guess for the linear velocity - "distance" relation, it is wrong even if $R r(1+z)$ or $R r(1+z)^{1} /{ }^{2}$ (equations 4 or 5 ) are taken to be the "luminosity distance". The correct expression to all orders of $z$ in the expanding case (eq. 4) for the luminosity distance is given by equation ( 8 ), whose series expansion is

$$
\mathrm{R}_{\mathrm{o}} \mathrm{r}(1+\mathrm{z})=\frac{\mathrm{cz}}{\mathrm{H}_{\mathrm{O}}}\left[1-\frac{\mathrm{z}}{2}\left(1-\mathrm{q}_{\mathrm{o}}\right)+\mathrm{O}\left(\mathrm{z}^{2}\right)\right] \text {. }
$$

This differs from Hubble's supposition (eq. 9) except when $q_{0}=1$; $a$ highly decelerating case.

Mattig (1958) first called attention to this difference by deriving the exact formulation of the $\mathrm{m}(\mathrm{z})$ relation as 


$$
\mathrm{m}_{\mathrm{bol}}=5 \log \frac{1}{\mathrm{q}_{\mathrm{o}}^{2}}\left\{\mathrm{q}_{\mathrm{o}} \mathrm{z}+\left(\mathrm{q}_{\mathrm{o}}-1\right)\left[-1+\left(1+2 \mathrm{q}_{\mathrm{o}} \mathrm{z}\right)^{1 / 2}\right]\right\}-5 \log \mathrm{H}_{\mathrm{o}}+\mathrm{M}
$$

whose series expansion, known to Heckmann (1942, which he derived from a model-independent Taylor series in $\dot{R}_{0}$ and $\stackrel{R}{0}_{0}$ using the light travel time as the independent variable) is

$$
\mathrm{m}=\text { const }+5 \log \mathrm{z}+1.086\left(1-\mathrm{q}_{\mathrm{o}}\right) \mathrm{z}+--\cdot
$$

Note that in the zero acceleration case of $q_{0}=0$, the expected $m(z)$ relation is $\sim 5 \log z+1.086 z$, rather than merely $5 \log z$.

The chief cosmological program for the 200 inch telescope, aside from correcting the distance scale, developed into obtaining reliable magnitude and redshift data to test equation (14), with the (unrealized)

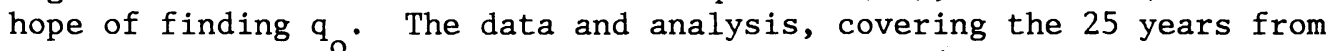
1950 to 1975, is described in a series of papers (Sandage 1968, 1972b,c, 1975a,b; Sandage and Hardy 1973; Gunn and Oke 1975; Kristian et al. 1978, Sandage and Tammannn 1983, 1986) whose final conclusion has been that the $\mathrm{m}(\mathrm{z})$ test is spoiled by the lack of precise knowledge of the evolutionary change, $\mathrm{dM} / \mathrm{dt}$, of the absolute magnitude of the standard candles with time (Sandage $1961 \mathrm{~b}$; Tinsley 1980 with references to her many detailed earlier papers on the effect). Again, Baade's dictum applies -- one cannot obtain $\mathrm{q}_{\mathrm{g}}$ until we know how $\mathrm{m}$ changes with time, i.e. until we understand how the stellar content of galaxies evolves.

It is fair to say that the present status of the work is that the velocity -- distance relation fits the linear case well to $z \sim 0.5$, and that deviations from $\log z \sim 0.2 \mathrm{~m}$ for $\mathrm{z}>0.5$ can be interpreted either as due (1) to a value of $q_{0}$ in the range $0.5 \lesssim q_{0} \lesssim 2$, or (2) to true smal1 departures from linearity for small $z$, for example as if $1+z \sim \exp$ $\mathrm{Hr}$ (see Pecker in this volume), or (3) to the combined effects of $q_{0} \neq 1$ and a luminosity evolution, $\ell(t)$, with time.

In this week we shall hear of the modern studies of the $\mathrm{m}(\mathrm{z})$ relation by Lilley and Longair (1984), Lilley et al. (1985), and by Spinrad (1986) and Djorgovski and Spinrad (1986) where these effects are discussed anew in the near infrared bandpass.

Finally, it is of interest to inquire into how universal is the redshift-distance relation, what is its scatter (intrinsic dispersion), and how close it can be traced to the Local Group.

To the extent it can be measured, the effect is universal, and isotropic to within small limits. Gross deviations from the Hubble flow, although occasionally claimed (Arp 1967, 1980; Burbidge 1981) are controversial -- not yet established.

These are, however, claims of "large" perturbations on the ideal Hubble flow ( $\mathrm{cf}$. Davies, this conference), but no claim of this kind has been made for systemic deviations larger than $\Delta v / v \sim 15 \%$. This is the limit that is still possible from the observed scatter in the $\mathrm{m}(\mathrm{z})$ relation for first ranked cluster and group galaxies (Sandage 1972b, 1975b; Sandage and Hardy 1973). Therefore, even if the recent claims 
(cf. Davies ${ }_{1}$ et $\underline{a l}$, opt. cit.) of deviations of $\sim 600 \mathrm{~km} \mathrm{~s}^{-1}$ for 〈cz〉 $\sim 4000 \mathrm{~km} \mathrm{~s}^{-1}$ are confirmed, the linear Hubble velocity-distance relation (with then local perturbations of $\lesssim 15 \%$ ) holds.

Finally, the linear expansion can be traced to within $\sim 2 \mathrm{Mpc}$ of the Galaxy using precise radio redshift data and distances determined largely from Cepheid variables. The relation is shown in Figure 5 . The dispersion about the linear relation is small at $\sigma(\mathrm{v}) \lesssim 60 \mathrm{~km} \mathrm{~s}^{-1}$, indicating a very quiet local Hubble flow.

Fig. 5 Modern data for the very local velocity-distance relation (Sandage 1986).
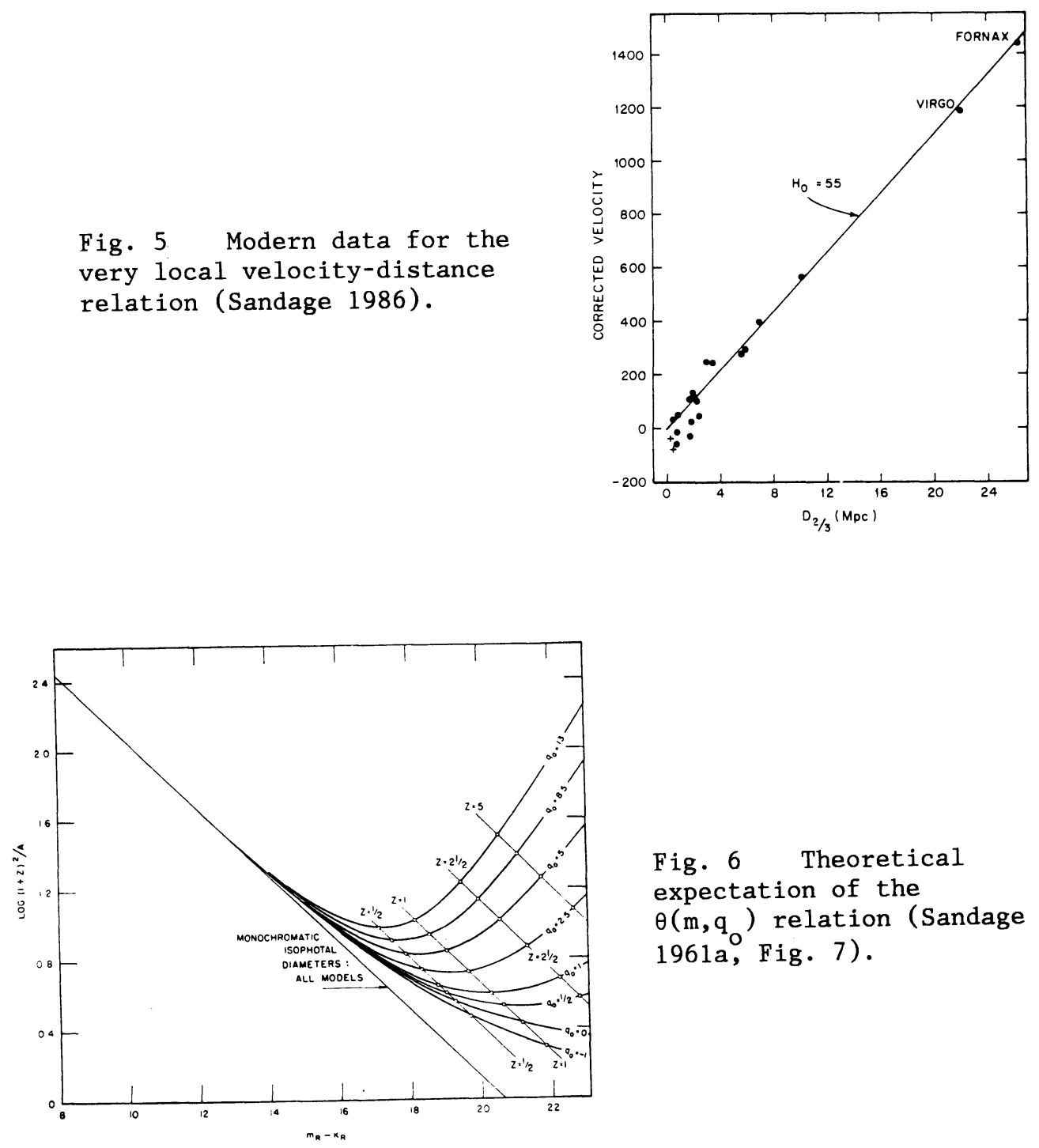

Fig. 6 Theoretical expectation of the $\theta\left(\mathrm{m}, \mathrm{q}_{\mathrm{o}}\right)$ relation (Sandage 1961a, Fig. 7). 
c) The angular diameter-redshift test was not proposed by Hubble, but by Hoyle (1959), where he showed that $\theta(z)$ has a dependence on $q_{0}$. The dependence of the corresponding $\theta(\mathrm{m})$ relation on $q_{0}$ for standard candles is shown in Figure 6 . The $\theta(z)$ diagram for standard meter sticks follows from the $\mathrm{m}\left(\mathrm{z}, \mathrm{q}_{\mathrm{o}}\right)$ relation of equation (14). Figure 6 shows that the metric diameters are predicted to decrease more slowly than $\theta \sim z^{-1}$ (depending on $q_{0}$ ), whereas isophotal diameters (i.e. angular sizes to a given isophote) are degenerate in $q_{0}$. However, the $\theta(z)$ isophotal relation is not so degenerate, but forms a family, giving, in principle another way to find $q_{0}$, provided that $\theta$ does not evolve with time.

The $\theta(z)$ isophotal relation for first ranked cluster galaxies is known to vary closely as $z$. Early optical data using eye estimates of $\theta$ (isophotal) are shown in Figure 7 .

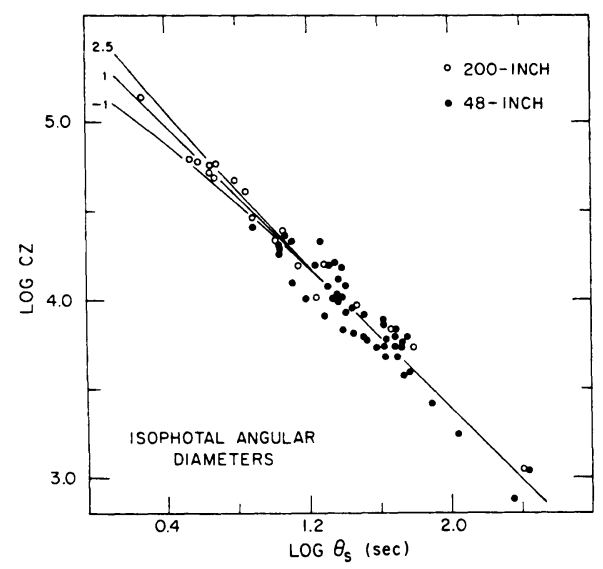

Fig. 7. Observed $\theta(z)$ relation for a first ranked cluster galaxies (Sandage 1972a).

This is, of course, a very strong test of the linearity of the velocitydistance relation, but it would be equivalent to the $m(z)$ Hubble diagram test if the surface brightness was strictly constant among first ranked cluster galaxies.

Fig. 8 Measured relation between metric diameter and redshift for first ranked cluster galaxies (Djorgovski and Spinrad 1981, their Fig. 3).

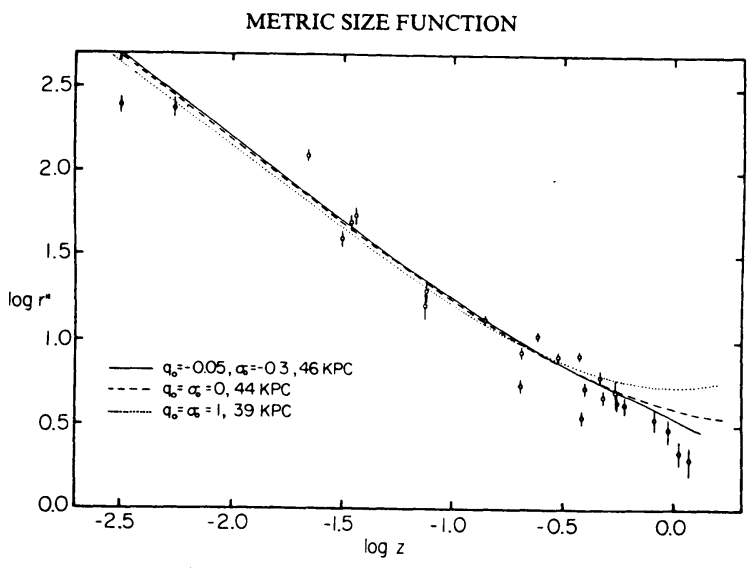


The current status of the metric diameter test is shown in Figure 8 from measurements of Djorgovski and Spinrad (1981) using $\theta$ (metric) for first ranked cluster galaxies as defined by Petrosian (1976). There is no indication of the expected turn-up in $\theta$ for large $z$. This can either be interpreted as an evolutionary change in the metric size with time, or as a proof that the theory is simply wrong. The point is crucial because it shows the circularity of the argument in each of the tests discussed so far. The observational fact is that the predictions of the Friedman models are not confirmed in detail in either the $N(\mathrm{~m})$, the $\mathrm{m}(\mathrm{z})$, or the $\theta(\mathrm{z})$ tests using the data as they are directly measured. In each case, to save the phenomenon, one imposes evolution of $\mathrm{m}$ or $\theta$ with time to force agreement and says, thereby, that one sees direct evidence for cosmic evolution with time. But it is clear that the conclusion would be correct only if we had independent knowledge that the standard Friedman cosmology is correct. This we do not have, being the object of the exercise itself. The arguments for each test, taken singly, are therefore circular. However, the case for the validity of the Friedman standard model and the consequent need for evolution corrections can be made in principle if all the available tests are used in concert and if the resulting evolution required by each makes a consistent evolutionary picture.

An important discussion of the consistency from such an approach is the parallel study of the angular size - flux density [ $\theta(\mathrm{S})]$ relation (Swarup 1975) and the number count - angular size [N( $\theta)]$ relation (Kapahi 1975) for radio sources over a wide range of flux density, suggesting indeed that evolutionary has occurred.

The $\theta(z)$ test was applied early to radio sources (Miley 1968, 1971; Legg 1970) using the angular separation of the double radio lobes. The test had been used earlier to show that, if radio galaxies were at their redshift distance, so then were quasars (Ryle 1968). Figure 9 shows Miley's $1971 \theta(z)$ diagram where the lack of the expected turn-up in $\theta$ for large $z$ is evident, violating the model predictions.

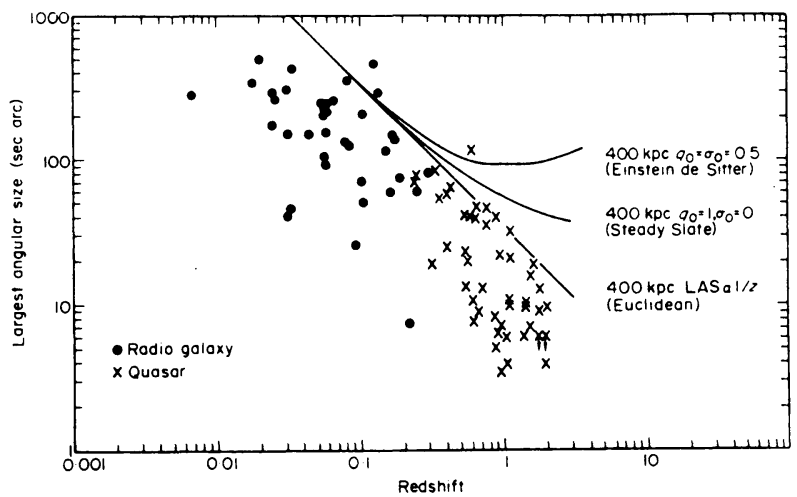

Fig. 9 The $\theta(z)$ relation for double lobed radio galaxies and quasars (Miley 1971).

However, in this case there is a very strong selection effect caused by the flux limited radio sample. This particular case serves as a good 
example by which to introduce the problem of selection effects in general, which we now discuss.

\section{SELECTION EFFECTS}

This week we shall repeatedly hear discussions of selection effects as they bias various results. Many of the effects are variations of the well known Malmquist bias which, in its simplest form, states that in a flux (apparent magnitude) limited sample, the average absolute luminosity of the nearby members of the sample is fainter than that which holds for more distant members. Stated that way, the effect is obvious, but the consequences of this $\langle M(z)\rangle$ variation in flux limited samples are profound. In every data sample where the objects have a non-zero dispersion, $\sigma(M)$, in absolute luminosity, the bias must be accounted for. The purpose of this section is merely to illustrate the effect with two examples.

The bias as it relates specifically to $\mathrm{H}_{\mathrm{O}}$ will often be discussed in the sessions that follow. This most crucial case is not discussed here as a third example, except to say the obvious that each side of the $\mathrm{H}$ controversy believes that their data have been properly corrected for selection effects, while those of their opponents have not. It remains to be seen if the explanation of the present discrepancy by a factor of two in $\mathrm{H}_{0}$ is, indeed, caused by selection effects.

\section{a) Bias in the radio data:}

When large numbers of redshifts became available for identified radio galaxies and quasars, and when the absolute radio power radiated between the rest-frequencies of $10^{7}$ and $10^{10} \mathrm{~Hz}$ was calculated for each (assuming $q_{0}=+1$ ), it was found that the radio power $L_{R}$ increased nearly precisely as $z^{2}$ for the available sample. The conclusion was not, of course, that the average radio power for the radio sources did, in fact, increase as $z^{2}$, but rather that the sample was severely restricted by the apparent flux limit of the catalogs, which was between $\sim 9$ and $3 \mathrm{Jy}$. Because the radio luminosity function $\phi(L)$ is so broad, covering at least a factor of $10^{6}$ in $L_{R}$, the observed effect is entirely the Malmquist bias.

Figure 10 shows the data, using mostly 3C sources. The 3C catalog has an apparent flux limit $9 \mathrm{Jy}$ at $178 \mathrm{MHz}$. Lines of constant apparent flux at 10,3 , and $1 \mathrm{Jy}$ are sloped with $\mathrm{a} \sim \mathrm{z}^{2}$ dependence in Figure 10. The distance modulii, $\mathrm{m}-\mathrm{M}$, are calculated from the redshifts, using $\mathrm{H}=50$. The lack of data points in the upper left is due to the volume effect, coupled with the very steep $\phi\left(\mathrm{L}_{R}\right)$ at the bright absolute radio power end.

Figure 10 shows the method by which any sample can be tested for a bias of this kind. One plots absolute magnitude (or $10 g$ L) vs $10 g$ distance for objects which have had these values calculated by any algorithm (eg. brightest stars, redshift, total apparent magnitude, 
Tully-Fisher etc. as the distance indicator). If there is a trend of $\langle M\rangle$ with distance, then either the effect is real, or one is sampling only a decreasing fraction of the total spread in $L$ as the distance is increased. The test is infallible. It should be applied to all samples as an indication of either freedom from Malmquist, or, very rarely, a real effect.

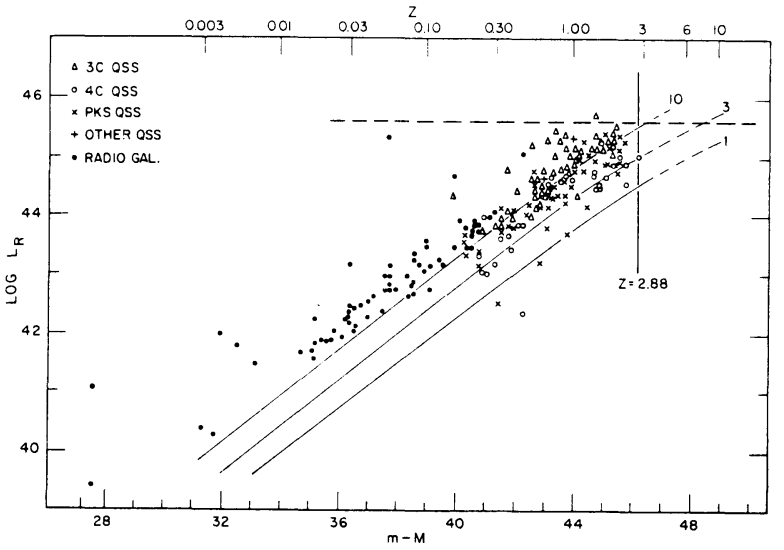

Fig. 10 Correlation between the absolute radio power, $\mathrm{L}_{\mathrm{R}}$, between $10^{7}$ and $10^{10}$ $\mathrm{Hz}$ in proper wavelength, and the distance modulus calculated from the redshift-distance relation. Lines of constant apparent flux density at 10,3 , and $1 \mathrm{Jz}$ are shown (Sandage 1972c).

Figure 10 is an extreme example because the $3 \mathrm{C}$ catalog contains only the apparently brightest radio sources. Only very nearby radio galaxies of low absolute radio power can enter the catalog. For the same reason, there are no $M$ dwarf stars in the Bright Star Catalog, reaching as it does only to apparent magnitude of $\mathrm{V} \sim 7$. The correlation in Figure 10 shows that the galaxies in Miley's diagram (Figure 9 here) at large redshift have much higher absolute radio power than those at low redshift, by about $\left(z_{1} / z_{2}\right)^{2}$. If, then, the linear separation of double lobed radio sources were to be a function of $\mathrm{L}_{R}$ (from the unknown physics), one would not be comparing a standard meter stick at different distances in Figure 9, but rather one that itself stretched or shrunk in linear diameter, with $z$. This would then provide no cosmological test. Much will be said this week about ways to test for such true physical effects (eg. especially Kapahi in this volume).

\section{b) Example of bias in optically selected data:}

Figure 11 is equivalent to Figure 10 but is a simulation adapted from Spaenhauer (1978), showing in the upper panel the distribution of absolute magnitudes in a volume limited sample, assuming $\langle M\rangle=-18.0$ and $\sigma(M)=2 \mathrm{mag}$. The lower panel is that part of the upper panel that is available in a magnitude limited sample, cut at apparent magnitude $\mathrm{m}=$ 13. The mean absolute magnitude at any distance in such a biased sample is clearly a function of distance.

The effect of more luminous galaxies at larger distances in a magnitude limited sample is strongly present in the Shapley-Ames galaxy sample, shown in Figure 12, which shows the absolute magnitude 


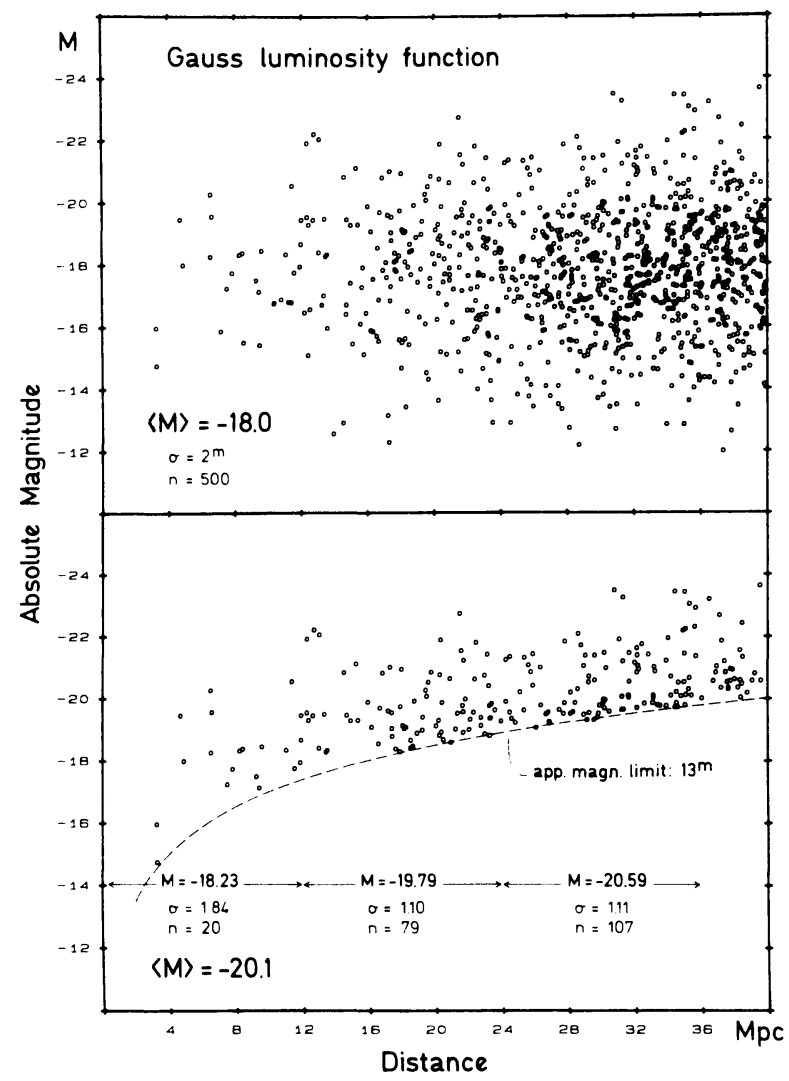

Fig. 11 Spaenhauer's simulation illustrating the Malmquist bias for a sample of galaxies whose volume limited mean magnitude is $\langle\mathrm{M}\rangle=-18$, with an intrinsic dispersion of $\sigma(M)=2 \mathrm{mag}$. The apparent magnitude limit of the catalog at $\mathrm{m}=13$ is shown in the lower panel.

(calculated from a non-perturbed Hubble flow using $\mathrm{H}_{0}=50$, together with the measured redshift) vs $10 \mathrm{~g}$ redshift for all $\mathrm{E}$ and ${ }^{\circ} \mathrm{S} 0$ Shapley-Ames galaxies with $|\mathrm{b}|>30^{\circ}$, excluding the Fornax and the Virgo Cluster fields. Figure 13 shows that the variation of $\left\langle M_{B}\right\rangle$ with distance is the expected Malmquist bias. The lower limit line is for a cut-off magnitude of $m_{B}=13.2$. The upper curved envelope is the calculated volume effect using the standard galaxy luminosity function. The fact that the observed data are contained within the two calculated limit lines shows directly the Malmquist bias.

The data for spirals (Tammann, et al. 1979) in Figure 14 again shows the strong Malmquist effect, indicating that these galaxies have a broad luminosity function. It has been claimed that part of the effect in Figures $11,12,13$, and 14 is caused by an error due to the assumption_of an unperturbed Hubble flow locally - to distances of $\sim 2000 \mathrm{~km} \mathrm{~s}^{-1}$. However, calculations using any reasonable local perturbation on the Hubble flow (Kraan-Korteweg et al. 1984) show the same pattern, indicating that $\phi(M)$ is broad, and that the Malmquist effect biases all discussions of field galaxy correlations in magnitude limited spaces.

Without correction for this bias, the apparent Hubble constant 


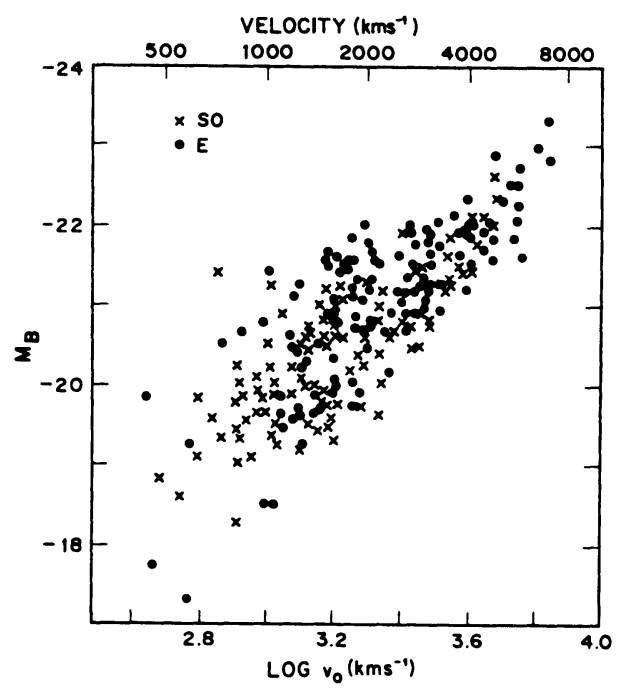

Fig. 12 The observed correlation of $M_{B}$ and redshift for $E$ and $S 0$ galaxies in the Shapley-Ames catalog. The strong increase of mean luminosity with increasing velocity is a direct indication of the Malmquist bias in the Shapley-Ames bright galaxy list. (Diagram from Sandage et al. 1979).

Fig. 13 Same as Figure 12 but with the calculated limit lines superposed as if the entire correlation was due to magnitude limited sample with $m_{\text {limit }}=13.2$ and a normal luminosity function with which to calculate the upper volume effect curved envelope (same reference as for Fig. 12).

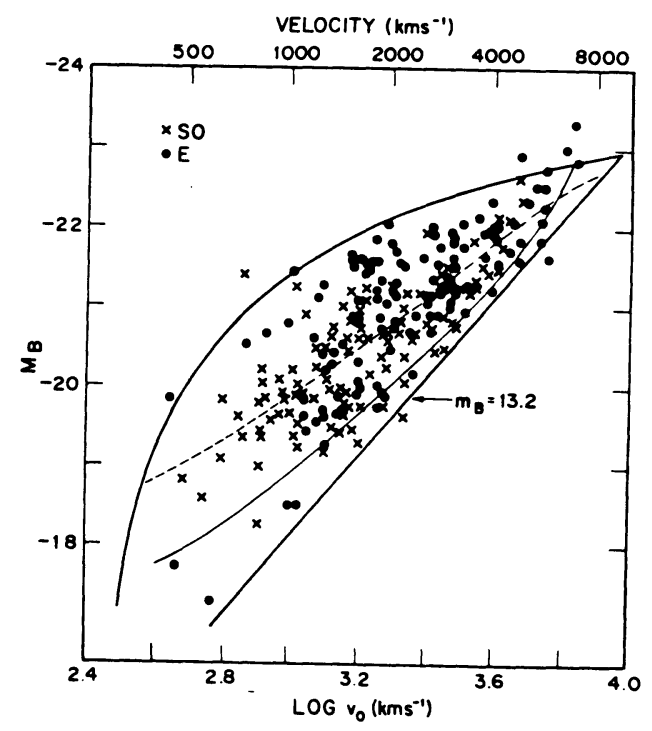

would appear to increase with distance, simply because incorrect faint values for $\langle M\rangle$ would be assumed for the more distant galaxies, making their calculated distances smaller than their real distances - the effect increasing with increasing distances (de Vaucouleurs and Peters 1986, their Figs $2 a$ and $2 b$ ). 


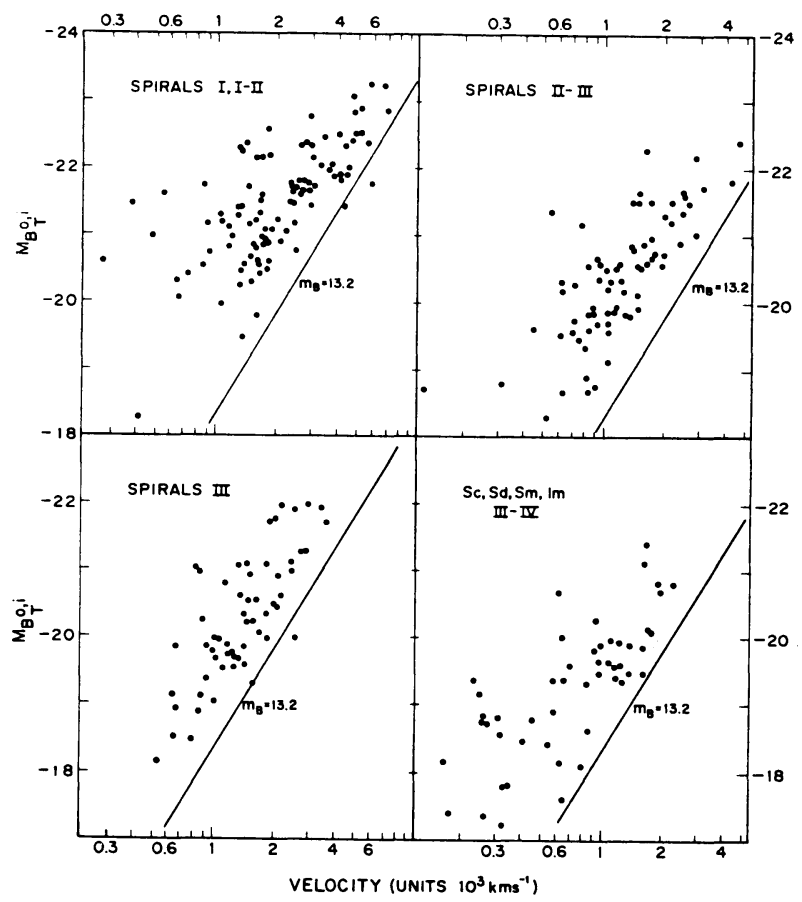

Fig. 14 The correlation of absolute blue magnitude with velocity for Shapley-Ames spirals of various van den Bergh luminosity classes illustrating the presence of the strong Malmquist bias in this sample. (Diagram from Tammann et al. 1979).

THE TIME SCALE TEST

The most powerful test concerns the various time scales; powerful because it is independent of the evolutionary problems that effect the $\mathrm{N}(\mathrm{m}), \mathrm{N}(\mathrm{z}), \mathrm{m}(\mathrm{z}), \underline{\theta}(\mathrm{m})$, and $\theta(z)$ relations. The test is to compare the time found from $\mathrm{H}^{-}$with an independent determination of the age of the Universe from the age of globular clusters, increased by the gestation period of the Galaxy after the creation event.

The age of Galactic globular clusters, based on the luminosity of their main sequence turn-offs, determined by the requirements of the Oosterhoff effect for the relative level of the horizontal branches (Sandage et al. 1981; Sandage 1981, 1982), is

$$
\mathrm{T}_{\circledast}=17 \pm 3 \times 10^{9} \text { years }
$$

This assumes $M_{V}(R R, M 3)=0.6$ and $M_{V}(R R, M 15)=0.8$, consistent with the requirements of the Oosterhoff effect, and zero-pointed by trigonometric parallaxes of field subdwarfs of low metal abundance (Sandage 1970, 1982).

The intrinsic geometry of the Universe, charafterized by the value of $q_{0}$ could be found by comparing the value of $\mathrm{H}_{0}{ }^{-1}$ with the age of the Universe $\mathrm{T}_{0}$, if both were known, by using the $\mathrm{T}_{0}{ }_{0}=\mathrm{H}_{0}{ }^{-\mathrm{F}}\left(\mathrm{q}_{\mathrm{o}}, \Lambda\right)$ relations (Sandage 1961a, Eqs. 61,62,65). 
The present situation is that $\mathrm{H}^{-1}$ is not agreed upon to within a factor of 2. The consequence of this disagreement are so well known as to requife no additional comment here, except to set out that, if $\mathrm{H}_{0}=$ $50 \mathrm{~km} \mathrm{~s}_{-1} \mathrm{Mpc}^{-1}$, then $\mathrm{H}^{-1}(50)=19.5 \times 10^{9}$ years, and that if $\mathrm{H}_{\mathrm{O}}={ }^{\mathrm{O}} 100$ then $\mathrm{H}^{-1}\left(100=9.8 \times 10^{9}\right.$ years.

If $\mathrm{q}_{\mathrm{O}}=0$, then $\mathrm{T}_{\mathrm{O}}=\mathrm{H}_{\mathrm{O}}^{-1}$. Hence equation (16) could be satisfied if $\mathrm{H}_{1}=50$ and $\mathrm{q}_{\mathrm{O}} \simeq 0$. OHowever, if $\mathrm{q}_{\mathrm{O}}=1 / 2\left(\right.$ or $\left.\Omega_{\mathrm{o}}=1\right)$ then $\mathrm{T}=2 / 3 \mathrm{H}_{\mathrm{O}}$ $=13^{\circ} \times 10^{9}$ years for $\mathrm{H}_{\mathrm{O}}=50$, and $6.5 \times 10^{9}$ years for $\mathrm{H}_{0}=100$. Neither case would satisfy equation (16) if $\Lambda=0$.

A consistent solution is for $\Lambda=0, \mathrm{H}=50$ (or $\mathrm{H}^{-1}=19.5 \times 10^{9}$ years), and $\mathrm{T}_{0}=\mathrm{T}_{\otimes}$ (from eq. 16) $+2.5 \times 10^{9}$ years for the age of the Universe, assuming $2.5 \times 10^{9} \mathrm{yr}$ as the gestation time for galaxies, This puts the epoch of galaxy formation at $\mathrm{z} \sim 7$. My own belief is that $\mathrm{H}_{0}=$ 42. All time-scale problems would then be solved if $q_{0} \sim 0.1$ and $\Lambda=0$.

Because the time scale test is independent of the types of evolutionary corrections that are necessary for the $m(z), \theta(m)$, and $\theta(z)$ tests, its importance cannot be overemphasized. It is for this reason that such a major effort must be expended to determine its value using powerful enough methods that the problem is placed beyond controversy. This has not yet happened. I doubt if any of the present methods are powerful enough to instill that kind of universal acceptance today, and I believe that no amount of further argumentation by any of the present groups, using the same methods that are yet so controversial, will solve the problem. It was in this vain that I suggested to Longair (quoted in his summary) that similar argumentation in the future along the same paths as used in the past, will not relieve the dilemma, and hence that a moratorium on the present arguments be called. But, not for the moment do I believe the solution to the problem should be called off only that new methods are needed by new groups whose advocacy positions are not involved.

\section{EVOLUTION IN THE LOOK BACK TIME}

We shall hear very much about attempts to find evidence for evolution of galaxy properties in the look-back time. Such effects must be present in an evolving Universe. Failure to detect them would lead to the important conclusion that a creation event did not occur. Unequivocal evidence that the effects are present would be the Ur proof that a creation event did occur.

At present, the evidence for the existence of such evolutionary differences (with $z$ ) is marginal. Direct evidence for changes of spectral energy distributions (Wilkinson and Oke 1978) and of colors (Kristian et al. 1978) show no measurable evolution to redshifts of 0.5, but none is expected over this short look-back time.

The most direct test for some sort of evolution of galaxies in clusters is that of Butcher and Oemler $(1984,1985)$ where they show (with rather high statistical significance) that the \% of blue cluster galaxies increases with $z$. This suggests a conversion process of star- 
forming galaxies into benign systems in the very short look-back times of $z<0.5$. Dressler and Gunn (1982) found evidence of a similar kind in the increased spectral peculiarity of cluster galaxies with increasing redshift. Neither of these results can be understood as passive evolution of stars in a given galaxy over so short a look-back time. The effects, if proved, must then be interpreted as some type of "interactive" evolution caused by the cluster environment. This type of work is expected to provide a dominant theme in the next decade.

Evidence for evolution of the Universe with time was found soon after work on the surface density of quasars as a function of apparent magnitude was begun. From even the first surveys it was found that $\mathrm{N}(\mathrm{m})$ increased more steeply than 0.6m (Sandage and Luyten 1967, 1969). Detailed work in the intervening years by the Bologna group (Braccasi et a1.), by the Wills (cf. this conference), by Usher and collaborators, Osmer and Smith, Arp, Schmidt and Green (1983 for a recent summary), and by many others has strengthened the data. There now seems little doubt that either density or luminosity evolution is required to explain the steepness of the $\mathrm{N}(\mathrm{m})$ quasar counts brighter than $\mathrm{B}=21$ and its rapid flattening at fainter levels. These data are generally considered to be the strongest evidence now available for look-back evolution.

Evidence for the direct evolution of the stellar content has been sought from changes in the spectra of $\mathrm{E}$ and $\mathrm{SO}$ galaxies over the redshift range of $0<z<1$. Although Wilkinson and Oke failed to find evidence in their sample to $z_{\sim} \sim 0.5$, Spinrad (1986) and Hamilton (1985) have searched for the effect on the $H$ and $K$ break near $\lambda 4000 \mathrm{~A}$ in spectra to $z^{\sim 0.8}$. Models by Bruzual (1983) predict a $\sim 15 \%$ effect at z 0.8. Models by Hamilton predict essentially no effect for $z<0.8$. The observations are contradictory. Spinrad observes the predicted $\sim 15 \%$. Hamilton observes none.

As this is a most direct test, the result is fundamental. Selection effects, operating differently on the different samples, are thought to be the explanation for part of the difference between Spinrad and Hamilton. It is expected that a large effort will be put on this and similar tests in the next decade to test for evolution.

\section{LARGE SCALE STRUCTURE}

Hubble (1934) was among the first to suggest large scale clustering properties. He did not observe the tendency to clump into sheets and nodes, as has now been done starting in $\sim 1975$, but rather he obtained a distribution of galaxy counts per unit area, reduced to standard observing conditions, that was a Gaussian normal distribution in $10 \mathrm{~g}$ $\mathrm{N}(\mathrm{m})$ rather than $\mathrm{N}(\mathrm{m})$. This is the distribution to be expected in contagion situations, rather than in conditions of independence. Hubble's data were convincing. Because the log normal form with the same dispersion was observed over the entire area of his survey, and because he obtained $\log \mathrm{N}(\mathrm{m}) \sim 0.6 \mathrm{~m}$ for $\mathrm{m}_{\sim}<19$, he concluded that the Universe was homogeneous on the largest scale that he could measure (over $\sim 2 \pi$ steradians and to a depth of $\mathrm{m} \sim 22$ ), but that it was clumped 
(i.e. clustered) on an intermediate scale. Hubble's evidence for the log normal distribution is shown in Figure 15, together with the figure caption to his diagram from Realm of the Nebulae.

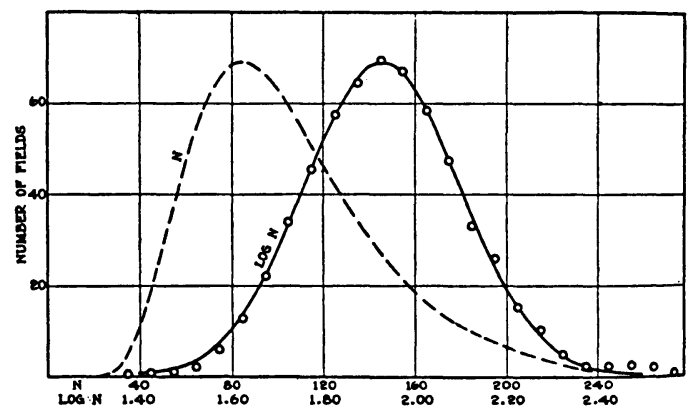

Fig. 7. Frequency-Distribution of Samples of Various Sizes.

The circles indicate numbers of samples in which the logarithms of the numbers of nebule bave various values. The smooth curve drawn through the circles is a normal error-curve. The close fit of the error-curve indicates that the $\log N$ per sample are distributed at random around the mean $\log N$ for all the samples.

When simple numbers of nebulæ per sample are substituted for the loga. rithms, the frequency distribution follows the unsymmetrical curve indicated by the dashes.
Fig. 15 Hubble's (1934) distribution of galaxy number counts from his survey of the number of galaxies per unit sky area in many areas. The distribution is a Gaussian in $\log \mathrm{N}$ rather than $\mathrm{N}$, indicating contagion. The diagram is Figure 7 in The Realm of the Nebulae.

A modern study by Crane and Saslaw (1986), using the Zwicky et al. catalogs, obtained the same result as Hubble, and drew the same conclusion.

The discovery of the filaments along which the galaxies concentrate is the major advance during the past decade. Much will be discussed during the week on this topic, with the review of the recent work by Geller (this volume). The subject began, in two dimensions, with a reanalysis of the classical galaxy catalogs in a series of papers by Peebles and his collaborators, with a parallel development by Einasto and his collaborators. The most striking result from the series of papers by Peebles et al. was an analysis of the Shane-Wirtanen counts which showed the filamentary nature of the distribution of galaxies brighter than $\mathrm{m}^{\sim 19}$ (Seldner et a1. 1977). The conclusions were unexpected, but confirmation from a different direction soon came using the addition of the third dimension as redshift space. The convincing data and powerful visualization was by Gregory and Thompson (1978, their Fig. 2). This paper marks the discovery of voids, which have become central to the subject. Prior work by Einasto et al. (1980 with earlier references), Tifft and Gregory (1976), and Chincarini and Rood (1976), foreshadowed the development, but the Gregory and Thompson discovery is generally recognized as the most convincing early demonstration.

Rapid developments in the mapping of various filaments and voids include the studies of Tarenghi et al. (1979), Gregory et al. (1981), Kirshner et al. (1981), Gregory and Thompson (1982), Chincarini et a1. (1983), and Huchra et al. (1983). A general review is given by Oort (1983).

Illustrations of the very rapid recent progress in deep mapping of particular regions will be given by Ellis and by Koo at this conference. 
Figures 16 and 17, from Haynes and Giovanelli's (1986) mapping of the Perseus region, show the richness of the present data.

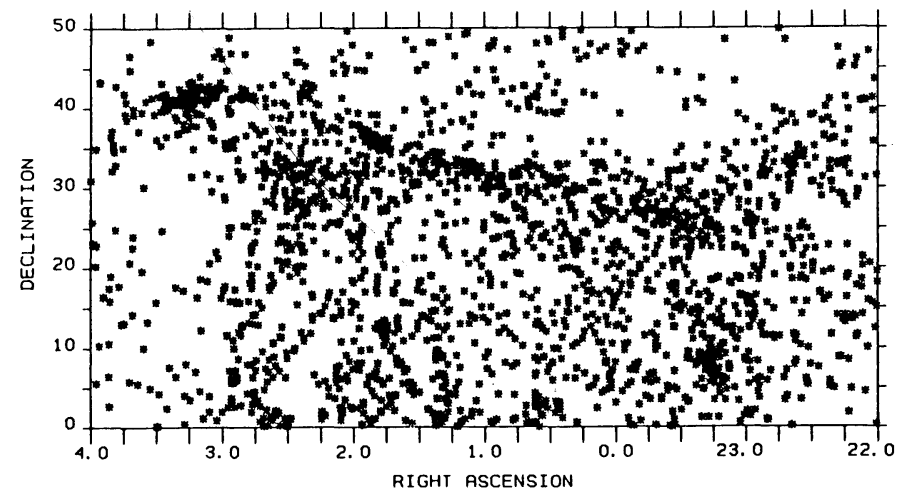

Fig. 16 The distribution in RA and Dec of galaxies in the Perseus region from the UGC catalog showing the projection of the Perseus filament on the plane of the sky. Diagram from Haynes and Giovane11i (1986).

Fig. 17 The $\pi$ diagram for galaxies in the same general direction as Figure 16. The presence of filaments and voids is typical of all other regions surveyed to date. The data and diagram is from Haynes and Giovane11i (1986).

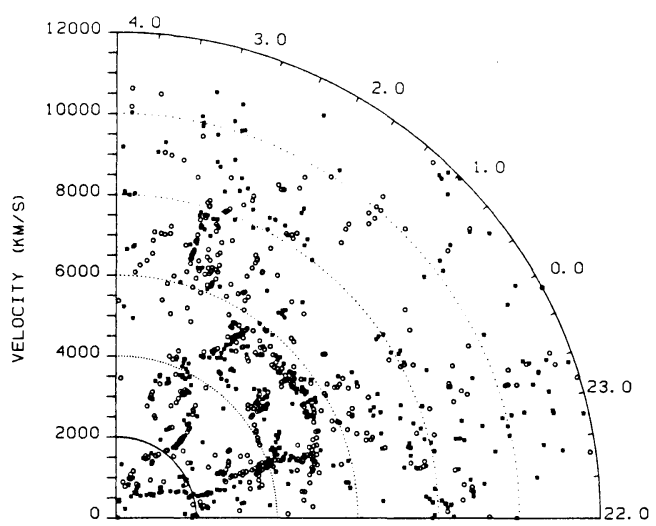

The $\pi$ diagram of Figure 17 is typical of the recent evidence for sheets and voids over scales of $\sim 200 \mathrm{Mpc}$. It is the projection of such structures on the plane of the sky that explains the filaments that are very clearly visible in the Shane-Wirtanen Lick counts (Seldner et al. 1977) and, for example, in Figure 16.

Theories of the production of the voids and sheets, following the original ideas of Zeldovich for pancake structures, have dominated the recent theoretical literature on galaxy and supergalaxy formation. These are to be reviewed by Dekel and others at this meeting.

\section{OTHER TOPICS}

A principal subject of the conference is galaxy formation (Silk) and the related topics of the galaxy luminosity function and the properties of galaxies along the Hubble sequence. Hubble began both of the latter suhjects in his papers between 1920 and 1936 . The work was later systematized by the concept of stellar populations within his. scheme of galaxy classification. 
Little of that will be discussed at this conference, although the variation of galaxy properties along the Hubble classification sequence undoubtedly holds clues to galaxy formation and evolution.

\section{THE NEXT TWO DECADES}

Finally, one can set out a list of unsolved problems for which the new large instrumentation will be adequate for solution. My list includes:

1) Proof or not that the redshift is a true expansion via the surface brightness test that $\mathrm{SB} \sim(1+z)^{-4}$ for a true expansion, $S B \sim(1+z)^{-1}$ for some form of tired light. For this, it is crucial that a metric diameter be used, probably adopting Petrosian's (1976) suggestion for its form. Although the tired light hypothesis is unlikely theoretically on the basis of the non-observed spreading of the images with increasing redshift, and the non-widening of the spectral lines, also with increasing $z$, the test should be made. Until now, attempts have been unsuccessful due to lack of a properly defined metric diameter.

2) Proof or not of evolution of the galaxy stellar content in the look-back time. The variation of the $\mathrm{H}$ and $\mathrm{K}, \lambda 4000 \mathrm{~A}$ break with $\mathrm{z}$ would seem to be the likely experiment, following Spinrad and Hamilton using larger data samples and a larger redshift range.

3) The most important experiment to test the Friedman model and/or the value of $q_{0}$ (with a possible need for $\Lambda \neq 0$ ) is the value of $H_{0}$ compared with the globular cluster time scale.

4) The compatibility of the clustering properties (filaments and "small" scale voids and inhomogeneities) with possible variations of the Hubble flow, following the bias introduced in the Hubble diagram by such inhomogeneities (Sandage and Tammann 1975, Appendix; Fall and Jones 1976). The necessary large scale redshift surveys that will be completed in this period should provide the data by which to model the problem.

5) Studies of the galaxy luminosity functions for different galaxy types and for the extremes of the density environments such as in the rich cluster cores and in the voids. Do dwarf galaxies exist in the voids such as is expected in certain models of biased galaxy formation?

6) The necessity to detect the $\delta \mathrm{T} / \mathrm{T}$ fluctuations of the $3^{\circ} \mathrm{K}$ radiation at a level of one part in $\sim 10^{5.5}$ on small angular scales. A failure to detect such fluctuations (presumed to be necessary for galaxy formation) would cause one to wonder if the Friedman standard model is correct. If not, the subject might again be back near its first beginnings. 


\section{ACKNOWLEDGEMENTS}

Part of the work on galaxies and observational cosmology at Mount Wilson was supported by National Science Foundation grant AST 82-15063 for which I am grateful. It is a pleasure to thank Janet Krupsaw for the preparation of the many drafts of this review with skill and patience.

\section{DISCUSSION}

Burke: In the spoken version of your talk, you set out a list of 12 great cosmologists of the 1930's, six observers and six theoreticians. The observers were Lundmark, Lindblad, Hubble, Humason, Baade, and Mayall. The theoreticians were Friedman, Lemaitre, de Sitter, Tolman, Robertson, and Gamow. To bring your list to 15 would you accept Einstein and Weyl on the theorist list, and V. M. Slipher among the observers?

Sandage: My list of 12 was meant to be evocative rather than definitive. Your choices are, of course, fascinating. Why are they not on my list? With hindsight, Slipher is often mentioned as having found the expansion. It has never been clear that this was understood in 21925 . He did not publish his redshift values, nor did he discuss them. He sent them to Strömberg who discussed them circa 1924 and to Eddington who published them in 21925. Without a discussion, are isolated measurements a basis to change the World which the discovery of the linear velocity-distance relation for all galaxies has, in fact, done?

Einstein: In the presence of God one does not mention God. It was his equations to which Friedman found a particular expanding solution. Nevertheless, we all learned cosmology not by reading Friedman but rather we teach our students from the Newtonian analogs of the equations first set out by Milne and McCrea. Their influence and that of G. C. McVittie has been paramount in making the subject understandable to more ordinary minds. Their names should have been added to the first hurried list.

Rubin: You discussed the effect of the Malmquist bias on the velocity studies, but are there not equivalent effects on the spatial plots, i.e. the strings in the distributions that are now showing up. Won't a bias due to such local non-homogeneities be present from that effect?

Sandage: Indeed. Both Fall and Jones (1976) and Tammann and I (1975) suggested that apparent deviations from the Hubble flow need not be real but would appear in the analysis of the $\mathrm{m}(\mathrm{z})$ relation for ScI galaxies (for example), caused by the bias effect on the data sample by a non-homogeneous spatial distribution of galaxies such as you suggest. 
Pecker: What about the age of the globular clusters? Quite possibly, turbulent diffusion, as suggested by Maeder and Schatzman might slow down the stellar evolution, and push to $25 \mathrm{Gyr}$. the age of globular clusters. This would add a severe difficulty to the modeling of the universal cosmology.

Sandage: The Friedman cosmology is already in difficulty (if $\Lambda=0$ ) unless $\mathrm{H}_{\mathrm{o}} \sim 50 \mathrm{~km} \mathrm{~s}^{-1} \mathrm{Mpc}^{-1}$, or less. Tammann and my current value from the supernovae observations of $\mathrm{m}(\mathrm{z})$ and with $M_{B}=-$ 20.0 at maximum gives $\mathrm{H}_{0}=42 \mathrm{~km} \mathrm{~s}^{-1} \mathrm{Mpc}^{-1}$. This can be fit with a $\Lambda=0, \mathrm{q}^{\sim 0}, \mathrm{~T}_{\mathrm{U}}=20 \times 10^{9}$ year data set. But, of course, I must agree with you that the longer the age scale of globular clusters is set and the shorter one puts $\mathrm{H}^{-1}$ (i.e. the larger one puts the value of $\mathrm{H}_{0}$ ), then the more you squeeze yourself out of the Friedman box.

Narlikar: I think that correct scientific practice requires that those who measure $\mathrm{H}$ are not influenced (indirectly) by what answers the ages of globular clusters give. Likewise those who estimate globular cluster ages do not look over their shoulders to see what answers the cosmologists want. After the two determinations have been made independently then the answers can be compared to check whether our cosmological picture is right. I hope this procedure is followed in the next twenty years.

Sandage: I believe, in fact, that it has been scrupulously followed in the past 20 as well.

Giraud: The variation of the Hubble constant that you have shown in a diagram by de Vaucouleurs and Peters is in the first few Mpc. How can you explain that by a Malmquist bias?

Sandage: The claim by de Vaucouleurs and Peters of a local variation of $\mathrm{H}_{\mathrm{O}}$ is over $\sim 40 \mathrm{Mpc}--$ more precisely it is over at least two times the distance to the Vifgo cluster (i.e. for observed velocities of $0<\sim<2000 \mathrm{~km} \mathrm{~s})$. This is precisely the range over which the very strong Malmquist bias exists shown in Figures 12, 13 and 14 of the written text for the Shapley-Ames spirals.

Audouze: In your introduction you did not mention the nucleochronology techniques to determine the age of the Universe. I am aware that these methods are still very uncertain (maybe more uncertain than the globular cluster technique), but we may hope in the future to obtain the age of the Universe from "chemical" (meteoritical) determinations. 
Sandage: Is it true that the present range of nucleosynthesis ages is from $\sim 9$ to $22 \mathrm{Gyr}$, depending on the assumptions of how the heavy element yield has changed with time - i.e. whether "sudden synthesis" or "gradual" enrichment of the ISM has occurred? I believe the globular cluster ages are accurate to $\sim \pm 25 \%$ and that the time between the creation event and the formation of globular clusters is accurately known from the high redshift cut-off of the quasar distribution at $z \sim 4$, signalling the first creation of the galaxies.

Norman: If one finds non-evolving galaxies at say $z \sim 1$ (for example as indicated by Hamilton's work, although the following statement is not dependent on this result), then they will be the oldest objects. Surely they should be the focus of our efforts to find $T_{0}$, not the globular clusters. One unambiguous nonevolving galaxy at redshift greater than $z=1$ would be a fundamental discovery and within it would contain the value of $\mathrm{T}_{\mathrm{O}}$

Sandage: The problem then becomes, how do you age date that galaxy? The only technique is to divine the stellar content by some type of spectral synthesis, and from it determine the main sequence termination point of the oldest stellar component. This is precisely the same technique as used for globular clusters except, for them, the main sequence termination luminosity is observed directly, and is therefore a more precise determination.

\section{REFERENCES}

Arp, H. C. 1967, Ap. J. 148, 321.

Arp, H. C. 1980 in Ninth Texas Symposium on Relativistic Astrophysics, ed. J. Ehlers, J. Perry, M. Walker: New York Academy of Sciences, p. 94 .

Brown, G. S., and Tinsley, B. M. 1974, Ap. J. 194, 555.

Bruzual, A. G. 1983, Ap. J. 273, 105.

Burbidge, G. R. 1981 in Tenth Texas Symposium on Relativistic

Astrophysics, ed. R. Ramaty and F. C. Jones: New York Academy of Sciences, p. 123.

Butcher, H. R., and Oemler, A. 1984, Ap. J. 285, 426.

Butcher, H. R., and Oemler, A. 1985, Ap. J. Suppl. 57, 665.

Chincarini, G. L., Giovane11, R., and Haynes, M. P. 1983, Ap. J. 269 13.

Chincarini, G., and Rood, H. J. 1976, Ap. J. 206, 30 .

Crane, P., and Saslaw, W. C. 1986, Ap. J. 301, 1 .

de Vaucouleurs, G., and Peters, W. L. 1986, Ap. J. 303, 19.

Djorgovski, S., and Spinrad, H. 1981, Ap. J. 251, $4 \overline{17}$. 
Djorgovski, S., and Spinrad, H. 1986, Ap. J., in press.

Dressler, A., and Gunn, J. E. 1982, Ap. J. 263, 533.

Einasto, J., Joeveer, M., and Saar, E. 1980, M.N.R.A.S., 193, 353.

Fal1, S. M., and Jones, B. J. T. 1976, Nature, 262, 457.

Gregory, S. A., and Thompson, L. A. 1978, Ap. J., 222, 784.

Gregory, S. A., and Thompson, L. A. 1982, Ap. J. 286, 422.

Gregory, S. A., and Thompson, L. A., and Tifft, W. G. 1981, Ap. J., 2433, 411.

Gunn, J. E., and Oke, J. B. 1975, Ap. J. 1995, 255.

Hamilton, D. 1985, Ap. J., 297, 371 .

Haynes, M. P., and Giovanelî, R. 1986, Ap. J. Lett., 306

Hoyle, F. 1959 in Paris Symposium on Radio Astronomy, ed: R. N.

Bracewe11, Stanford Univ. Press, p. 529.

Hubble, E. 1934, Ap. J., 79, 8.

Hubble, E. 1936, Realm of the Nebulae, Yale University Press.

Hubble, E. 1953, M.N.R.A.S., 113 658 .

Huchra, J., Davis, M., Latham, D., and Tonry, J. 1983, Ap. J. Supp1., $\underline{\underline{52}}, 89$.

Kapahi, V. K. 1975, M.N.R.A.S., 172, 513.

Karachentsev, I. D., and Kopylov, A. I. 1978, IAU Symp., 79, 339.

Kirshner, R. P., Oemler, A., Schechter, P. L., and Schectman, S. A. 1981, Ap. J. Lett., 248, L57.

Kraan-Korteweg, R. C., Sandage, A., and Tammann, G. A. 1984, Ap. J., 283,24 .

Kristian, J., Sandage, A., and Westpha1, J. A. 1978, Ap. J., 221, 383.

Kron, R. G. 1980, Ap. J. Supp1., 43, 305.

Legg, T. H. 1970, Nature, 226, 65.

Lilley, S. J., Longair, M. S. 1984, M.N.R.A.S., 211, 833.

Lilley, S. J., Longair, M. S., and Allington-Smith, J. R. 1985, M.N.R.A.S., 215, 37 .

Mattig, W. 1958, Astron. Nach., 284, 109.

Mattig, W. 1959, Astron. Nach., 28

Miley, G. 1968, Nature, 218, 933.

Miley, G. 1971, M.N.R.A.S., 157, 477.

Murdock, H. S., Hunstead, R. W., Pettini, M., and Blades, J. C. 1986, Ap. J., 309, October 1 .

Oort, J. H. 1983, Annual Rev. Astron. Astrophys., 21, 373.

Peterson, B. A., Ellis, R. S., Kibblewhite, E. J., Bridgeland, M. T., Hooley, T., and Horne, D. 1979, Ap. J. Lett., 233, L109.

Petrosian, V. 1976, Ap. J. Lett., 209, L1; 210, L53.

Ryle, M. 1968 in Highlights of Astronomy, Vol. 1, ed. L. Perek, IAU publications: Reidel, p. 33.

Sandage, A. 1961a, Ap. J., 133, 313.

Sandage, A. 1961b, Ap. J., $13 \frac{134}{1}, 916$.

Sandage, A. 1968, Observatory, 88, 91 .

Sandage, A. 1970, Ap. J., 162, $\overline{\overline{8}} \overline{1} 1$.

Sandage, A. 1972a, Ap. J., $173,485$. 
Sandage, A. 1972b, Ap. J., 178, 1 .

Sandage, A. 1972c, Ap. J., $178,25$.

Sandage, A. 1975a in Galaxies and the Universe: ed. A. Sandage, M. Sandage, and J. Kristian: Univ. Chicago Press, p. 761.

Sandage, A. 1975b, Ap. J., 202, 563.

Sandage, A. 1981, Ap. J., $2 \overline{\overline{4} \overline{8}}, 161$.

Sandage, A. 1982, Ap. J., $2 \overline{5} \overline{2}, 553$.

Sandage, A. 1986, Ap. J., $30 \overline{7}, 1$.

Sandage, A., and Hardy, E. 1973, Ap. J., 183, 743.

Sandage, A., Katem, B., and Sandage, M. 1981, Ap. J. Supp1., 46, 41 .

Sandage, A., and Luyten, W. J. 1967, Ap. J., 148, 767.

Sandage, A., and Luyten, W. J. 1969, Ap. J., $1 \overline{15}, 913$.

Sandage, A., and Tammann, G. A. 1975, Ap. J., , $197,265$.

Sandage, A., and Tammann, G. A. 1983 in Large Scale Structure of the Universe, Cosmology and Fundamental Physics. First ESO-CERN

Conference ed. G. Setti and L. van Hove, p. 127.

Sandage, A., and Tammann, G. A. 1986 in Inner Space Outer Space, ed. E. W. Kolb, M. S. Turner, D. Lindley, K. Olive, and D. Steckel: Univ. of Chicago Press, p. 41.

Sandage, A., Tammann, G. A., and Yahil, A. 1979, Ap. J., 232, 352 .

Schmidt, M., and Green, R. F. 1983, Ap. J., 269, 352.

Seldner, M., Siebers, B., Groth, E. J., and Peebles, P. J. E. 1977, A. J. , 82, 249 .

Spaenhauer, A. M. 1978, Astron. Astrophys., 65. 313.

Spinrad, H. 1986, Pub. A.S.P., 98, 269.

Swarup, G. 1975, M.N.R.A.S., 172

Tarenghi, M., Tifft, W. G., Chincarini, G., Rood, H. J., and Thompson, L. A. 1979, Ap. J., 234, 793 .

Tammann, G. A., Yahil, A., and Sandage, A. 1979, Ap. J., 234, 775.

Tifft, W. G., and Gregory, S. A. 1976, Ap. J., 205, 696.

Tinsley, B. M. 1980 in Physical Cosmology: Les Houches summer school; session XXXII: ed. R. Balian, J. Audouze, and D. Schramm: North Holland, P. 161.

Tyson, J. A., and Jarvis, J. F. 1979, Ap. J. Lett., 230, L153.

Wilkinson, A., and Oke, J. B. 1978, Ap. J., 220, 376 . 